\title{
"Liking"' other Drivers' Behavior while Driving
}

$\begin{array}{ll}\text { Chao Wang } & \text { Jun Hu } \\ \text { Eindhoven University of } & \text { Eindhoven University of } \\ \text { Technology } & \text { Technology } \\ \text { P. O. Box 513 } & \text { P. O. Box 513 } \\ \text { 5600 MB Eindhoven NL } & 5600 \text { MB Eindhoven NL } \\ \text { ch.wang@tue.nl } & \text { j.hu@tue.nl }\end{array}$

Jacques Terken

Eindhoven University of

Technology

P. O. Box 513

5600 MB Eindhoven NL

j.m.b.terken@tue.nl

\begin{abstract}
Driving is a social activity: Drivers need to coordinate with each other to share the road infrastructure. The social relationship between drivers also influences the driving behavior. With everywhere available connectivity and the broad penetration of social network services, the relationship between drivers on the road may gain more transparency, enabling social information to pass through the steel shell of the cars and giving opportunities to reduce anonymity and strengthen empathy. In this paper, we introduce a concept that enables drivers to express their appreciation for other drivers' polite driving behavior through gestural interaction, in order to improve their driving behavior by social awareness.
\end{abstract}

\section{Author Keywords}

Natural user interface, C2C communication, Social computing, Gestural interaction, Augmented Reality

\section{ACM Classification Keywords}

D.3.3 Information interfaces and presentation: User

Interfaces - interaction styles.

\section{Introduction}

Usually, driving occurs in a situation where there are other drivers around. This means that drivers need to coordinate with each other to share the infrastructure [1]. But when drivers sit in "iron cages", there are only few communication channels to support the coordination. Recently, new opportunities for communication between drivers have emerged through everywhere available connectivity of Internet and the broad penetration of social network services. The 
relationship between drivers on the road may gain more transparency, enabling social information to pass through the steel shell of the cars and giving opportunities to reduce anonymity and strengthen empathy [2], and to eliminate boredom, loneliness and stress. In this study, we propose a system based on Driver to Driver communication and social network, enabling users to express appreciation of nearby driver's polite behavior on the road. We present a gesture based interaction system for this concept and the results of a user test on a prototype integrated in a driving simulator.

\section{"Liking" other drivers' behavior}

From a previous study in which thirty different "socia car" ideas were discussed with more than twenty people, the "Liking other drivers' behavior" emerged as one of the most promising concepts. The concept holds that "You can give other drivers a "Liking" or a "Disliking". More than half of the participants tended to only accept the "Liking" feature as they thought "Disliking" could be abused. This concept was then selected for further exploration in a driving simulator study.

\section{Interaction design}

The current in-vehicle human-machine interface (HMI) is shaped to operate traditional in-vehicle tasks such as navigation, entertainment and phone calls [2], But in our case, the HMI system must enable drivers to interact with surrounding cars to accomplish socia interaction. There is no current interaction system to operate in such scenarios. However, the gestural interaction of POI (point of interest) which recently has become a hot topic in the in-vehicle HMI research domain can provide many significant references[3] as it also requires direct interaction with the environment.

Pointing gesture
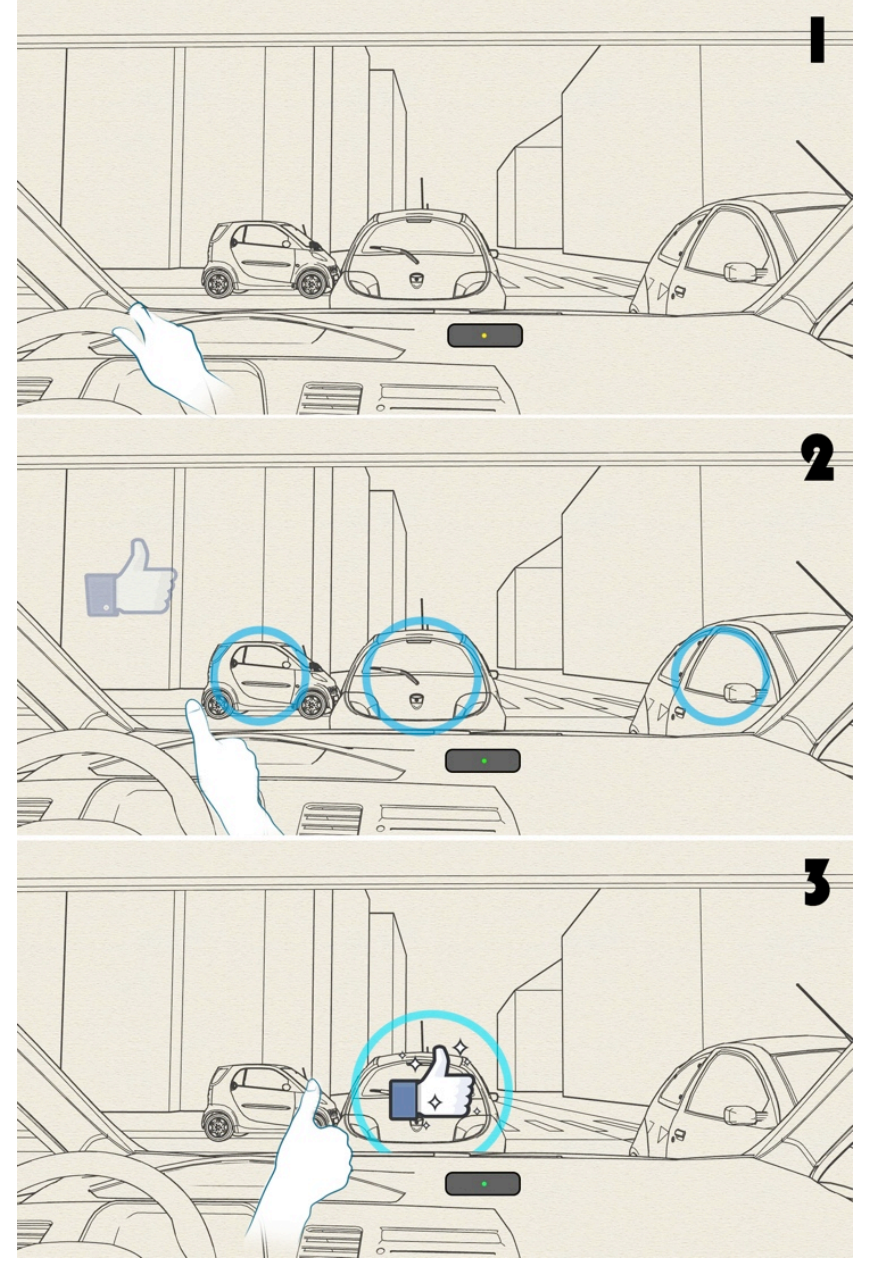

Figure 1. Procedures of "Liking" operation. Step 1: Normal driving status; Step 2: Stick out the thumb; Step 3: Point at the target and straighten arm. 


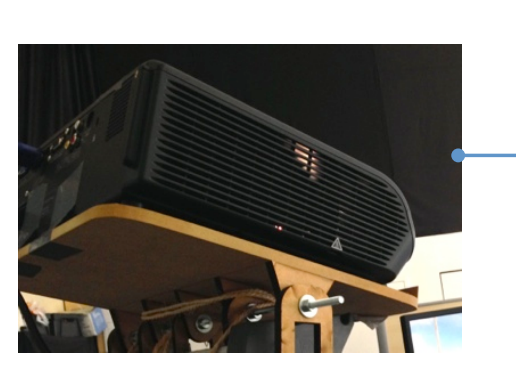

The high-power projector

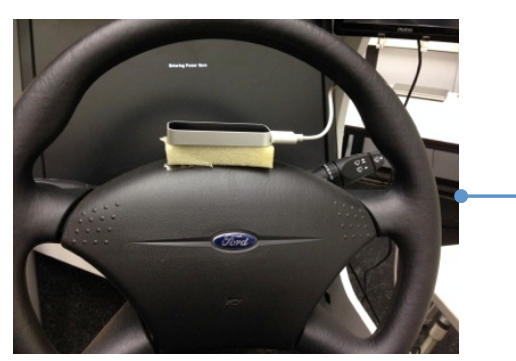

Leap motion fixed on the steering wheel

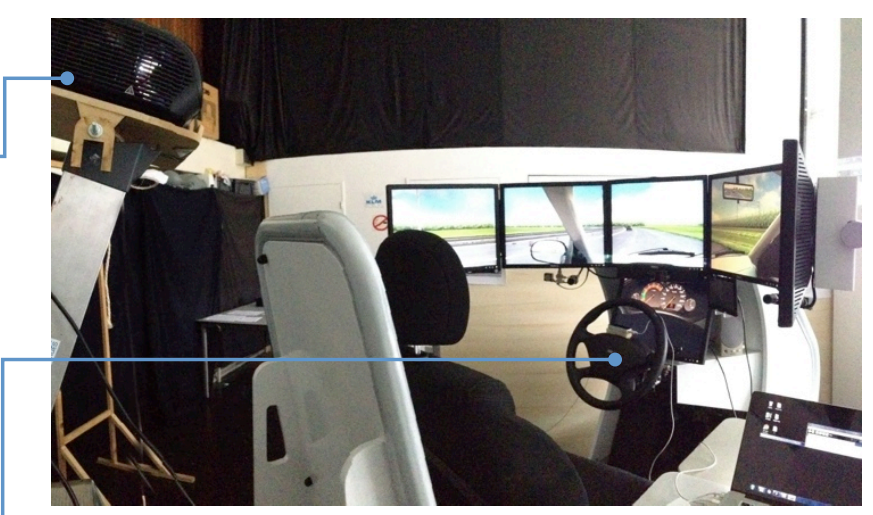

Figure 2. Setup of gestural interaction prototype based on driving simulator

The use of gestural interfaces for in-car interaction can reduce visual demand and thus increase safety [3]. The advantage of gesture is that it does not require physical interaction with another surface [4]. This topic has been researched on by academia and industry for many years. Althoff et al. [5] investigated 17 different hand gestures and six different head gestures, and used them for complex interactions with the in-car infotainment system to increase the expressive power of gestures. Mahr et al. [6] designed a reduced set of three micro-gestures, which could be applied while keeping the hands on the steering wheel. However, all of these approaches only replace existing controls in the menu structure. They do not introduce new use cases that only become possible with gestural interaction, such as the direct interaction with objects in the environment.

Gesture is a natural way to refer to physical items in the environment (deictic gestures) as well as a way to express spatial and physical relationships (iconic gestures) through relative hand positions and the trajectory of hand motions [7]. Rümelin et al. [8] investigated pointing as a lightweight form of gestural interaction in cars. They made a prototype that was able to recognize driver's pointing to objects outside the windshield by a depth camera. After a series of experiments, a recognition rate of $96 \%$ was achieved in the lab. In their system, the driver's hand had to remain in a stable position until the system confirmed the pointing operation by audio feedback. The results showed that the shorter the hold time, the higher is the tolerance of users. And feedback on the task of selecting an object in a street was positive. Fujimura et al. [4] proposed a system for driver interaction with the environment by enabling the driver to point to an outside target while maintaining a grasp on the steering wheel, combined with visual feedback in the form of a 3D Head-up Display (3D-HUD). However, their pointing gesture did not provide more semantic information than tip the objects in the environment. Further operations were needed to finish a concrete task.

Procedures of "Liking" operation

In our study, the main task of the application is to convey appreciation to a target car nearby. For this purpose, we designed an interaction flow which combined selecting target and conveying a "Liking" operation in one gesture, and give drivers clear visual feedback by an augmented reality display.

The flow of the "Liking" operation can be divided into 3 steps (Figure 1):

- Step 1: The driver is in the normal driving status. When the gesture sensor (possibly setup in the front of dash board) does not detect any gesture other than manipulating the car, the HUD is on standby or displays primary driving information such as speed and navigation.

- Step 2: The driver wants to give another person's driving behavior a "Liking". He releases one hand from the steeling wheel, sticks out the thumb to give the target car a "Liking". This activates a 


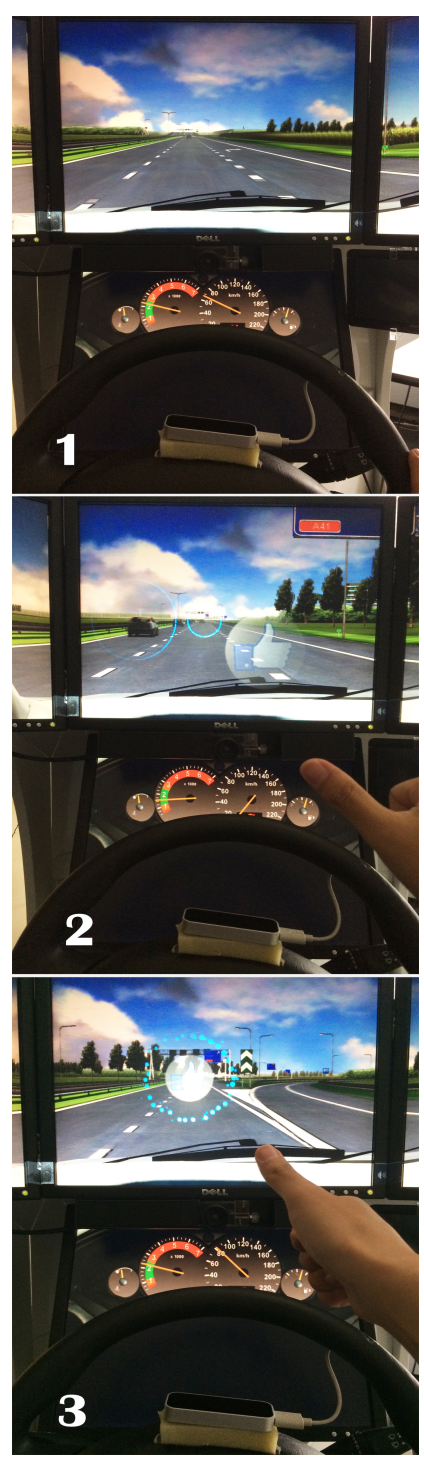

Figure 3. Implementation of design
Head-up Display (HUD), displaying several circles tagging cars nearby, and a thumb icon acting as a cursor to show his hand's relative position.

- Step 3: The driver point at the target car with his thumb and straightens his arm to confirm the "Liking" operation. The system recognizes this action and gives visual and audio feedback (see Fig. 3 panel 3).

\section{Apparatus}

A setup integrating a gestural sensor and projector with a driving simulator was created to evaluate the concept. The driving simulator system included steering wheel, seat, pedals, gears and six $17^{\prime \prime}$ screens (Figure 2)

In this study, we used a Leap Motion Controller, which is fixed on the steering wheel to track participants' gestures. The simulator computer transfers coordination data of the positions of nearby cars to an external laptop in real time, and the laptop projects the UI graphics directly on the simulator screen through a high-power projector. The application running on the laptop was developed using the Processing language. A video recorder was set behind the driver to record the whole process for further analysis. Figure 3 shows the 3 steps of the gestural interaction in the driving simulator.

\section{Experimental Design}

Ten participants ( 7 male, 3 female; ranging from 26 to 30 with mean 27.6, all with more than 5 years driving experience), took part in the test. Firstly, they were introduced to the topic of the study. Then, they received 15 minutes of training to get familiar with the system. The testing scenario was a straight highway with 3 lanes. They were asked to wave their hands, stick out their thumbs and give random cars a "Liking" while driving. After that, a bundle of tasks were given: 1 . The participants were required to follow a car whose speed was $110 \mathrm{~km} / \mathrm{h}$ on the highway and keep a distance of 250 meters. Then they were asked to change the distance to $200 \mathrm{~m}, 150 \mathrm{~m}, 100 \mathrm{~m}$ and give the front car 3 "Likings" respectively at these distances. 2 . They were asked to overtake the front car from the right lane. When they were approaching the slower car at the distance of 50 meters, they were asked to give him a "Liking". 3. They were ask to return back to the slow lane and wait for the overtaking of the behind car and give it a "Liking" at the distance of 30 meters. Each participant finished 5 rounds of tasks. Afterwards, a questionnaire capturing subjective workload, perceived usability and user experience was completed. And an interview was conducted to capture problems, preferences and general feedback.

\section{Results}

Subjective Workload

The subjective workload was assessed with the NASA TLX (Task Load Index) questionnaire. Ratings for all six dimensions were low to medium (Figure 4).

\section{Usability}

Perceived usability was assessed with the System Usability Scale (SUS). The total score was 73.5, which is "acceptable" to "good" comparing to average SUS scores [9]. The items "Do not need technical support" and "Did not need to learn a lot", which reflected learnability [10], gained a higher score than average $(P=0.068$ and 0.013$)$ (Figure 5$)$

\section{User Experience}

AttrakDiff [11] is helpful to evaluate the Hedonic quality (HQ) and Pragmatic quality (PQ) of this system. The results of the AttrakDiff questionnaire showed that the system was rated as fairly positive both in terms of hedonic and pragmatic quality (Figure 6). The overall impression of the product is very attractive, but there is room for improvement in terms of hedonic quality as well as usability. 


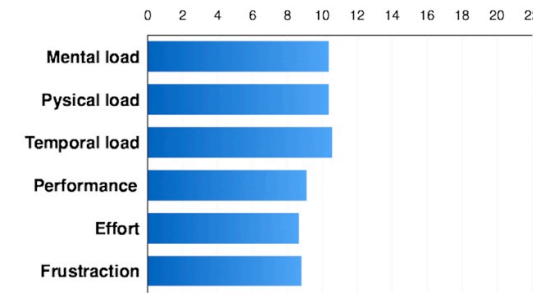

Figure 4. Subjective Workload (by NASA TLX questionnaire)

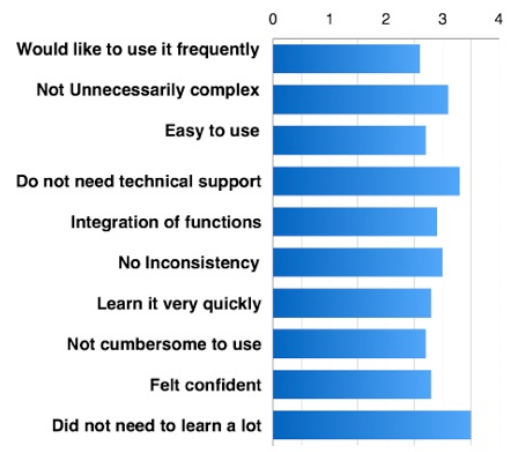

Figure 5. Perceived usability (by SUS)

\section{Discussion}

Evaluation of the concept

Participants evaluated the concept quite positively, as evident from the positive scores both for hedonic and pragmatic quality. Interestingly, more than half of the participants wanted to have a "Disliking" function added

Mapping gestures with visual feedback

Fujimura et al. divide the communication in their system (combined gesture and 3D-AR display) into two perspectives: Allowing the driver to input information and providing feedback to the driver. In our study, participants provided input by the "Liking" gesture, which was recognized by the Leap Motion sensor; feedback was provided by augmented reality displayed information, consisting of a "Liking" icon acting as a cursor which was projected on the simulator screen. Natural user interfaces combine input and output together. The most natural way is to remove the "Liking" cursor and enable driver to use hands to interact with other cars directly. However, that may cause another problem in a driving context: The focal plane of the user's thumb is different from the foca plane of the targets, which may increase visual distraction and decrease the accuracy of selection. That will be verified by other prototypes in future research.

Decreasing visual distraction

Most participants commented that the main source of distraction was the visual feedback. In this case, a particles effect was displayed to overlay the target car directly when a "Liking" operation succeeded (as shown in Fig. 3.3). Many participants complained that it blocked their sights. Some participants suggested moving the visual feedback to the top of the target car as the sight on the road should always be clear. Another important factor that was mentioned by 2 participants concerned the 2D graphical elements. The feedback information is a 2D graphic overlaying in a 3D world, which forced participants to "put" these elements into the scenario. After the test, we redesigned a more concise graphic effect of "Liking" feedback that contained 3D elements and was immersed into the scenario. The new visual design gained more poistive feedback when we demonstrated it to several participants.

Semantics and learnability

According to the statement of the participants, the "Liking" gesture was very intuitive and easy to remember. This was consistent with two items of the SUS questionnaire that could reflect learnability. Besides, they were also familiar with the thumb icon that was projected on the screen as it is widely used by social networking such as Facebook and Instagram. However, one participant argued that if there are many applications in the future, different gestures might be needed to control different applications, so that people might have to remember lots of different gestures. A better solution is that a single gesture might apply to several applications and the system might comprehend the semantics based on context. For instance, the system may not only be able to recognize sticking your thumb but also waving your hand to others as "Liking" operation. In other contexts, sticking your thumb and waving your hand might stand for "confirm" and "cancel" commands.

\section{Conclusion and future work}

In this study, we have evaluated a concept by which drivers may provide positive feedback about the driving behavior of fellow drivers. We found that the concept was appreciated by the participants, as evident from a positive score for hedonic quality. Also, we investigated gesture based interaction design for the concept. Investigation based on a small sample size showed that gestural interaction combined with augmented reality visual feedback, enabled drivers to express appreciation for others' driving behavior with limited workload and acceptable usability. 


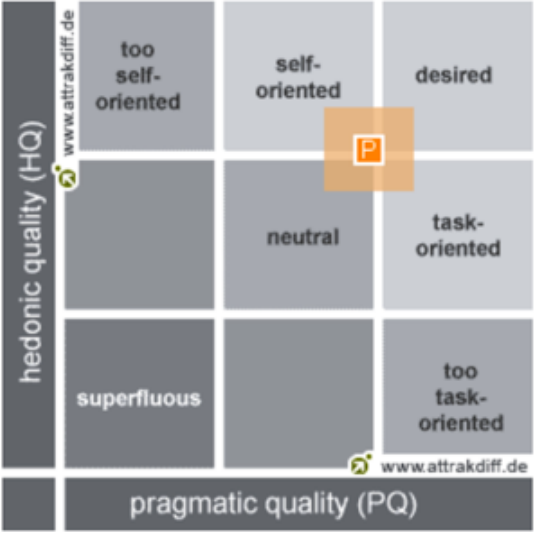

Figure 6. Result of user experience (by Attrakdiff)
We did not evaluate matters such as task completion time, error rate and visual distraction. Also, the influence on driving behavior was not analyzed and the sample size was small. Furthermore, the interaction is only one factor that influences the acceptance of the system. More importantly, being able to "Like" other drivers' behavior implies that, changing the perspective, our driving behavior may be "Liked" by other drivers. As a next step, we will change the perspective and investigate how drivers feel about receiving feedback evaluating their driving behavior from other drivers.

\section{References}

[1] O. Juhlin, Social Media on the Road. Springer Science \& Business Media, 2010.

[2] R. Schroeter, A. Rakotonirainy, and M. Foth "The social car: new interactive vehicular applications derived from social media and urban informatics," AUTO-UI '12, pp. 107-110, Oct. 2012.

[3] A. RIENER, "Gestural Interaction in Vehicular Applications," Computer, vol. 45, no. 4, pp. 42-47, 2012.

[4] K. Fujimura, L. Xu, C. Tran, R. Bhandari, and V. Ng-Thow-Hing, "Driver queries using wheel-constrained finger pointing and 3-D head-up display visual feedback," AUTO-UI '13, pp. 56-62, Oct. 2013.

[5] F. Althoff, R. Lindl, L. Walchshausl, and S Hoch, "Robust multimodal hand-and head gesture recognition for controlling automotive infotainment systems," VDI BERICHTE, 2005.

[6] A. Mahr, C. Endres, C. Müller, and T. Schneeberger, "Determining human-centered parameters of ergonomic micro-gesture interaction for drivers using the theater approach," AUTO-UI '11, pp. 151-158, Nov. 2011.

[7] D. McNeill, "Gesture and thought," NATO SECURITY THROUGH SCIENCE SERIES E ..., 2007.

[8] S. Rümelin, C. Marouane, and A. Butz, "Freehand pointing for identification and interaction with distant objects," AUTO-UI '13 pp. 40-47, Oct. 2013.

[9] A. Bangor, P. Kortum, and J. Miller,

"Determining what individual SUS scores mean: Adding an adjective rating scale," Journal of usability studies, 2009.

[10] J. R. Lewis and J. Sauro, "The Factor Structure of the System Usability Scale," vol. 5619, no. 12, pp. 94-103, 2009.

[11] M. Hassenzahl and A. Monk, "The inference of perceived usability from beauty," HumanComputer Interaction, 2010. 